\title{
Wavelet Transforms Applications and Interpretation Based on the Signal Genesis
}

\author{
S.Ananthi Arun, Ananthanarayanan ,S.Sivakannan ,G.Prashanth
}

\begin{abstract}
A signal from any measurement system provides insight into its genesis, thereby enabling an understanding of a certain activity or phenomenon. Seismic signals, radar echo signals, physiological signals, signals from specially fabricated instruments such as MRI, CT scanner all provide information by using an analysis that resolves the signal into its frequency components. While the Fourier transform and its fast evaluating algorithm known as FFT are standard for such analysis, there are presently additional signal transforms in use, of which "Wavelets" or Wavelet transform or wavelet decomposition are becoming very important. If the Fourier transform resolved the signal into its spectral components of Sine and Cosine waves, the Wavelets do the same in terms of non- sinusoidal oscillatory wave-shapes of burst - like appearance. This paper deals with the choice of wavelet transforms based on signal genesis and the interpretation required from the analysis of the signal, that one is expected to infer.
\end{abstract}

Keywords: Fourier Transform, Haar, Morlet. Daubechies Wavelet transform, Denoising, Data Compression.

\section{INTRODUCTION}

The exponentially decaying sinusoid is commonly the nature of response of all linear damped oscillatory systems such as a pendulum or spring - mass - viscosity system. In this case, a short time burst of an exponentially decaying sinusoid becomes a suitable wavelet. Understanding the existence of natural frequencies present in a system through the poles and zeros of the system function is the basis of Fourier transform methods. The spectral power density indicates the power of a signal at the various frequencies. The spectrogram is the graph, with time in the $\mathrm{X}$-axis, of the spectral power plotted at various frequencies along the $\mathrm{y}$-axis as an intensity plot. Given the Fourier transform of a signal it can be converted back to the signal in time do main through the inverse Fourier transform. The Fourier transform equations are well known.

Revised Manuscript Received on October 18, 2019.

Dr.S.Ananthi, Professor, Department of Electronics and Communication Engineering, MVJ College of Engineering Bangalore, Karnataka.

Dr Arun Ananthanarayanan, Assistant Professor, Department of Electronics and Communication Engineering, MVJ College of Engineering Bangalore, Karnataka.

Sivakannan Subramani, Assistant Professor, Department of Electronics and Communication Engineering, MVJ College of Engineering, Bengaluru, Karnataka

Mr.Prahanth, Assistant Professor, Department of Electronics and Communication Engineering, MVJ College of Engineering, Bangalore, Karnataka.

The wavelet transform is represented by alternative basis functions, of the term

$$
\begin{aligned}
& F(\omega)=\int_{-\infty}^{+\infty} f(t) \exp (-j \omega t) d t \\
& f(t)=\frac{1}{2 \pi} \int_{1}^{\infty} F(\omega) \exp (j \omega t) d \omega \\
& W(b, a)=\int \frac{1}{\sqrt{a}} \phi *(t-b / a) f(t) d t \ldots \text { (3) }
\end{aligned}
$$

which means the wavelet transform decomposes the signal $\mathrm{f}(\mathrm{t})$ into a set of scaled wavelet functions $\phi(t)$, which are real or complex as the case may be.

In [3], work on using wavelet decomposition of EEG raw signals with a view to compression and telemetering has been illustrated. The question of quantization of data samples, signal to noise ratio on wavelet compression has been shown in that paper. Today, with computer based EEG machines, these problems are solved already in the machine, since it produces acceptable data wave patterns on the screen and even directly networked data communication is feasible to the distant monitoring site. The use of wavelet compression is aimed only to reduce the number of data and to enable a quick investigation by a mere analysis of the co-efficients and to ease monitoring attention.

Signal patterns in EEG such as rhythms, wave and spikes must be properly de-compressed. Experimental data patterns were thus compressed and transmitted by PCM to a remote monitoring site. The nature of wavelet used was varied with the wave pattern from time to time, by choosing those wavelet coefficients that yielded the best fit for the signal under that time window with less number of coefficients. If theta rhythms were compressed using Morlet wavelets, the wave and spike patterns used a Daubechies-4 wavelet and so on. Sending that information also enables a quick decompression cum inference at the monitor. Over the long stretch of EEG records, the diagnostic inferences made with direct signal and the decompressed signal with the knowledge of what wavelet was used, were found to be very matching, and after some practice, a mere perusal of the wavelet pattern and the type of it enabled quick identification of the onset of disorders in the patient. In [3], work on using wavelet decomposition of EEG raw signals with a view to compression and telemetering has been

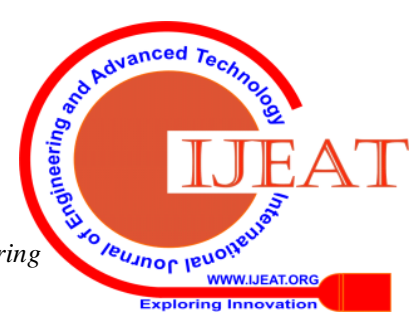




\section{Wavelet Transforms Applications and Interpretation Based On the Signal Genesis}

illustrated. The question of quantization of data samples, signal to noise ratio on wavelet compression has been shown in that paper. Today, with computer based EEG machines, these problems are solved already in the machine, since it produces acceptable data wave patterns on the screen and even directly networked data communication is feasible to the distant monitoring site. The use of wavelet compression is aimed only to reduce the number of data and to enable a quick investigation by a mere analysis of the co-efficients and to ease monitoring attention.

Further to the developments in signal analysis, the concept of Wavelet based signal decomposition has gained considerable ground in many areas of signal processing. The wavelet transform, much like the frequency analyzer, converts the signal over a length of time into a set of wavelet groups, which, when summed up, will be matching the signal without much error. If a period of E.E.G. activity that was considered as a pure "delta" rhythm, it was because the wave patterns that were noted had frequencies in the limited region associated with "delta" waves and thence that particular region of E.E.G. record was simply spelt as "Delta pattern observed." So much so, if a region of the E.E.G. record shows up to be somewhat more complex and is non determinable by the conventional rhythm patterns, then recourse may be made to use the Wavelet algorithm for time-frequency reconstruction of the signal of the record.

\section{TYPES OF WAVELETS FOR CHOICE}

Wavelet transform can be characterized as universal, orthogonal, flat, polynomial - regular or Fourier polynomial . If $\mathrm{A}$ is a wavelet matrix, the following equation called scaling equation is associated with

$$
A=\left(a_{k}^{s}\right) \text {. }
$$

The matrix has rank $m$ and genus $g$

$$
\phi(x)=\sum_{k=0}^{m g-1} a_{k}{ }^{0} \phi(m x-k)
$$

For e.g., the Harr matrices of rank 2 are :

$$
\left(\begin{array}{cc}
1 & 1 \\
1 & -1
\end{array}\right),\left(\begin{array}{cc}
1 & 1 \\
-1 & 1
\end{array}\right),\left(\begin{array}{cc}
1 & 1 \\
-e^{j \theta} & e^{j \theta}
\end{array}\right)
$$

The first of the above Haar matrices gives rise to the function $\phi$ as a rectangular wave. The second function called 'wavelet function' is denoted as $\psi$, and is given by the next row of the matrix in general,

$$
\begin{aligned}
& \left.a_{k}{ }^{s}\right) \text { as } \\
& \psi(x)=\sum_{k=0}^{m g-1} a_{k}^{1} \phi(m x-k)
\end{aligned}
$$
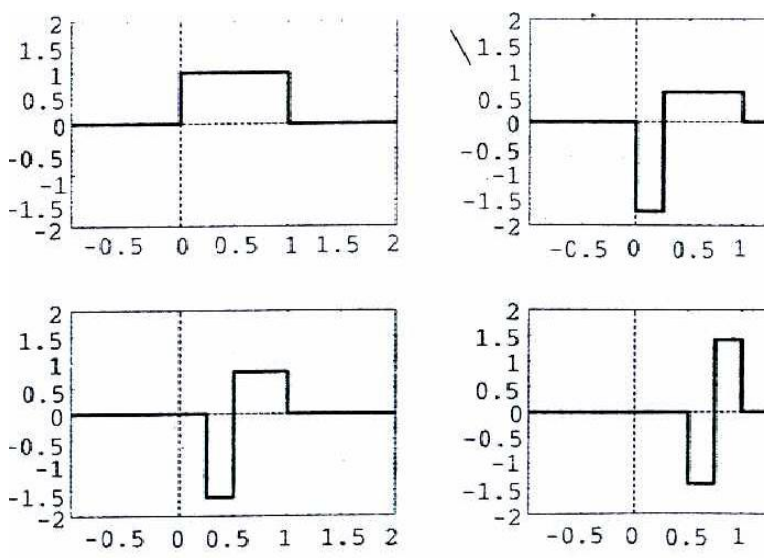

Fig.1. Shows Haar wavelet -Scaling and Wavelet Function

The discrete Fourier transform matrix (DFT) of rank $\mathrm{m}$ is based on the values of

$$
\left(\begin{array}{ccc}
1 & \cdots & 1 \\
& W^{*} & \\
1 & \cdots & W(=-1) 2
\end{array}\right)
$$

where $\mathrm{s}<\mathrm{m}$. This is also a Haar Matrix. If $\mathrm{m}=2$, it becomes

$$
\left(\begin{array}{cc}
1 & 1 \\
1 & -1
\end{array}\right)
$$

which is the matrix considered as the first canonical Haar matrix. The application of the Haar matrix based wavelet function of fig.1. simply uses two points of the input signal $\mathrm{s}(\mathrm{t})$, i.e., $\mathrm{sn}, \mathrm{sn}+1$ and the $\phi(x)$ finds the sum $(\mathrm{sn}+\mathrm{sn}+1) /$ 2 , while $\psi(x)$ finds the difference $(\mathrm{sn}-\mathrm{sn}+1) / 2$. These are just got by the terms 1,1 and $1,-1$ in this simple matrix. This is only a filtering process of the two - point signal, the sum giving a low - frequency filtering component and the difference giving the high frequency component. This leads to the ' filter bank' structure for finding the wavelet spectrum. There is a high pass filter $\mathrm{h} 1$, and a low pass filter h0, where they are related to scaling and wavelet functions by the relations;

$$
\begin{aligned}
& \phi(t)=\sqrt{2} \sum_{n} h_{0}(n) \phi(2 t-n) \\
& \psi_{i}(t)=\sqrt{2} \sum_{n} h_{i}(n) \phi(2 t-n)(i=1 \text { or } 2)
\end{aligned}
$$

It is necessary to subsample the output of these filters (by 2, taking every other sample) to avoid redundancy. Fig.2.a, b show how this process is carried out on

the input signal value and how by a further and further sub division into high and low part frequencies, the final wavelet multi-resolution decomposition of the input signal is obtained. Fig.2c shows the filter bank structure providing the analysis signals $\mathrm{cn}, \mathrm{dn}$ and how the synthesis with inverse filter functions can get back $y n=x n$ ( except for the sampling delay) exactly, (in the case of orthogonal wavelets).

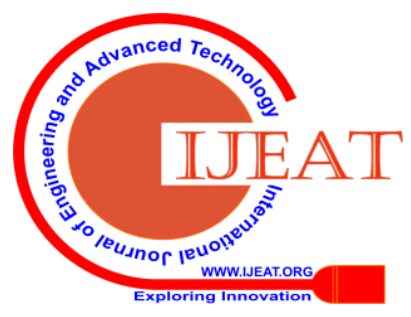




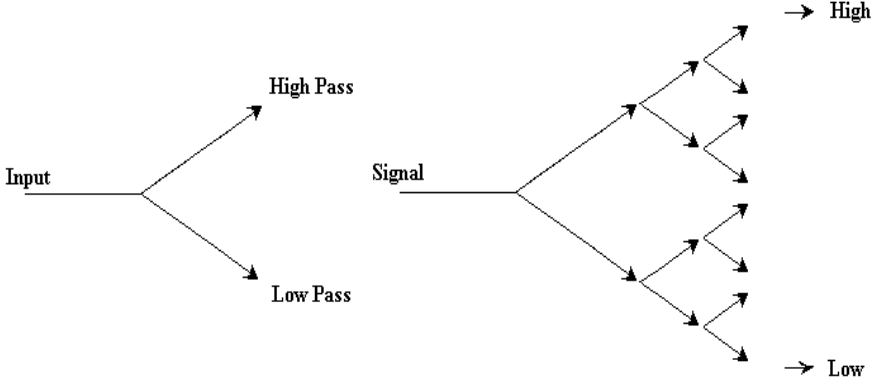

Fig.2a

Fig.2c

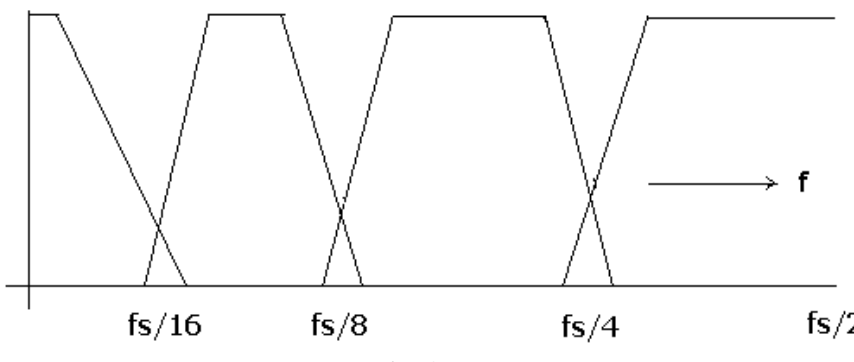

Fig.2d

The components of fig.2(b) cover the entire frequency range in steps of 2, logarithmically. This feature is similar to how musical notes are classified in several octaves of frequencies ( fig.2d). The subband coding scheme of DWT is also shown in fig. 3 .

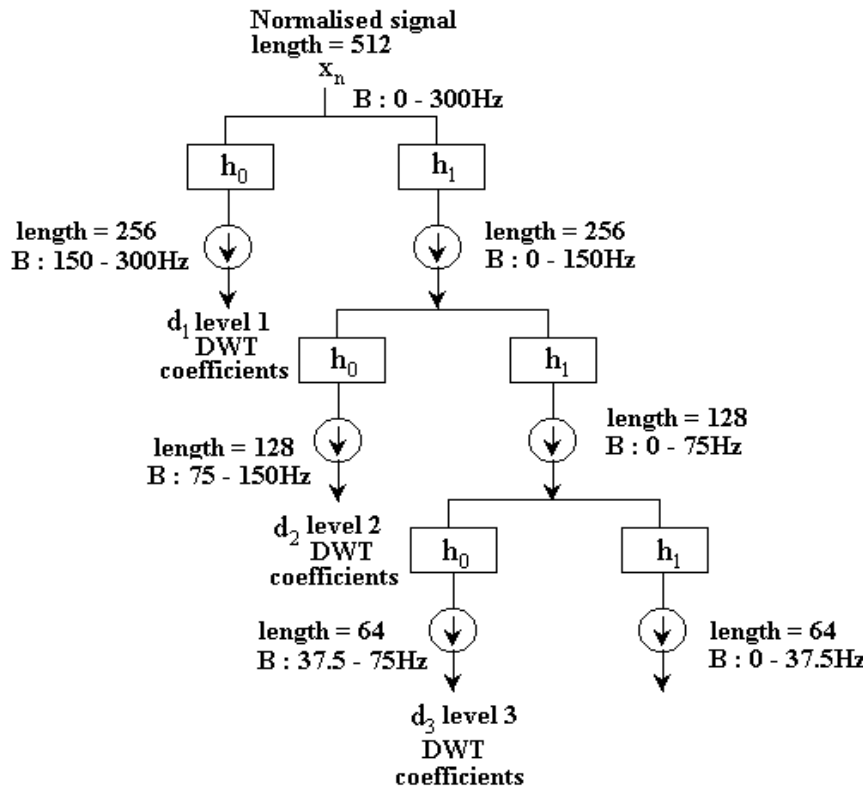

Fig.3. Subband DWT coding of a $300 \mathrm{~Hz}$ bandwidth signal. The upper levels of di contain fewer and fewer coefficients, due to sub-sampling.

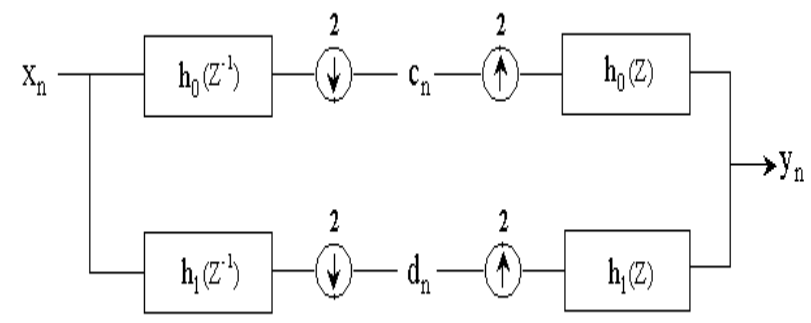

This kind of frequency scaling produces points in the timefrequency grid such that $m$ as the scale (or equivalent requency) rises by a factor 2 , the number of grid points get reduced by $1 / 2$. Such a ' critically sampled' DWT shown in fig.4 produces a ' scalogram' (similar to the FFT spectrogram) having sparsely located points. In order to improve this, a double density DWT using an over sampled analysis filter bank is employed, but they suffer with the difficulty in accurately re - synthesizing ( $y$ is not equal to xn ).

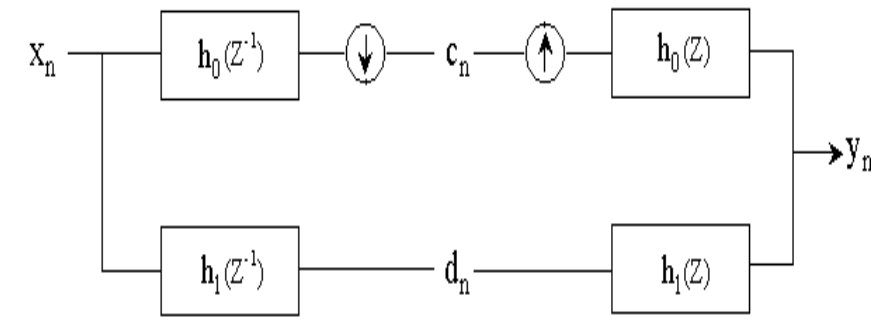

Fig. 4 a). Shows the Double density DWT

In this context of filters of wavelet analysis, it is necessary to understand the terms rank $r$, genus $g$ and matrix A. By rank (r), we mean the number of banks in a m-band filter bank. If the filter has rank $m$, it samples the signal $m$ times per unit time. $M=\infty$ means analog. The genus(g), of the transform represents the number of signaling intervals ( symbols) over which the filter operates. This $\mathrm{m}, \mathrm{g}$ denote the number of taps in each subband filter. Thus $\mathrm{A}$ is the chosen Haar matrix of $\mathrm{m} \mathrm{x} \mathrm{m}$.
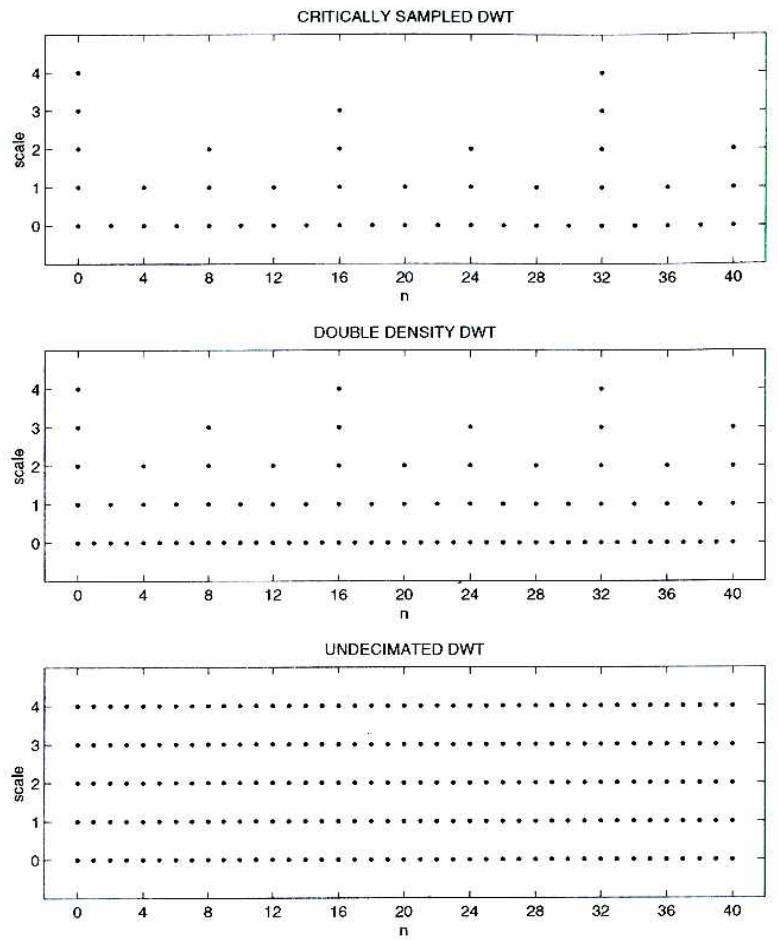

Fig.4. Points in the time - scale grid for three different methods.

A matrix of higher genus than rank $r$ introduces information from neighboring blocks. While increasing the rank corresponds to an increased resolution ( an FFT of rank 64 being twice as many frequency bins as an FFT of

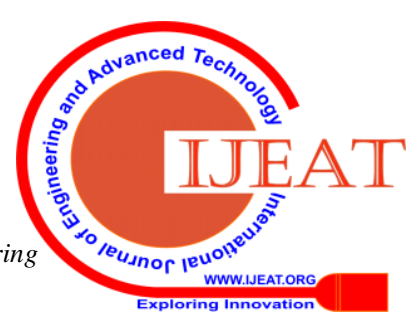




\section{Wavelet Transforms Applications and Interpretation Based On the Signal Genesis}

rank 32), genus increase does a greater overlapping of transform 'window'. Donbechie' s wavelet matrix of rank2 and genus 2 is given by

$$
D_{2}=\frac{1}{4}\left(\begin{array}{cccc}
1+\sqrt{3} & 3+\sqrt{3} & 3-\sqrt{3} & 1-\sqrt{3} \\
-1+\sqrt{3} & 3-\sqrt{3} & -3-\sqrt{3} & 1+\sqrt{3}
\end{array}\right) \ldots(11)
$$

Another wavelet matrix of rank 2 and genus 2 is

$$
A(\theta)=\left(\begin{array}{llll}
a_{0}(\theta) & a_{1}(\theta) & a_{2}(\theta) & a_{3}(\theta) \\
b_{0}(\theta) & b_{1}(\theta) & b_{2}(\theta) & b_{3}(\theta)
\end{array}\right) \ldots(12)
$$

where

$$
\begin{aligned}
& a_{0}(\theta)=0.5\left(1+\sqrt{2} \cos \left(\theta+45^{\circ}\right)\right) \\
& a_{1}(\theta)=0.5\left(1+\sqrt{2} \cos \left(\theta-45^{\circ}\right)\right) \\
& a_{2}(\theta)=0.5\left(1-\sqrt{2} \cos \left(\theta+45^{\circ}\right)\right) \\
& a_{3}(\theta)=0.5\left(1-\sqrt{2} \cos \left(\theta-45^{\circ}\right)\right)
\end{aligned}
$$

The associated $b(0)$ terms are

$$
b_{k}(\theta)=(-1)^{\kappa+1} a_{3-k}(\theta)
$$

For the Daubechie' s r $=2, \mathrm{~g}=2$ wavelet, the scaling and wavelet functions are as shown in fig.5.

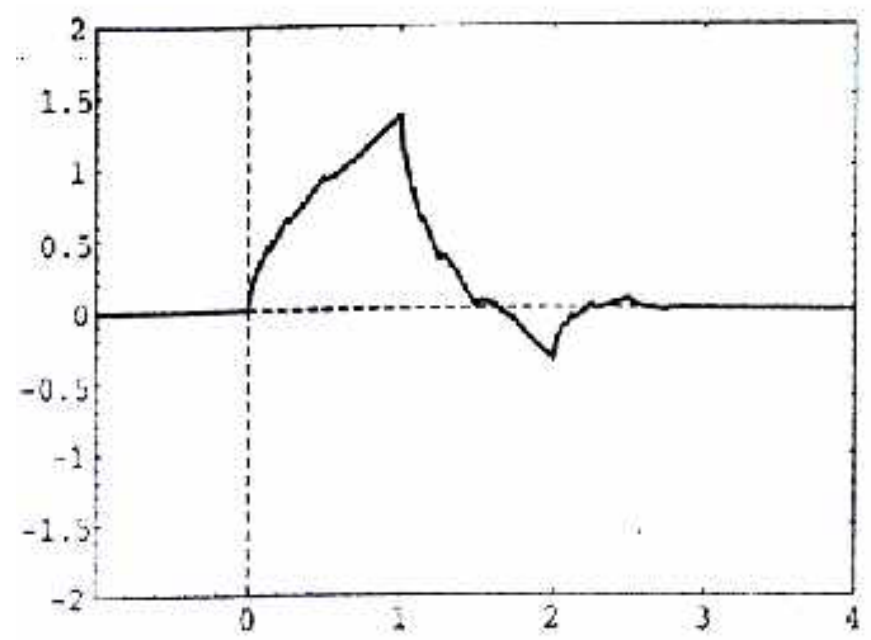

Fig.5a. Daubeches Scaling function (For Rank 2 and Genus 2)

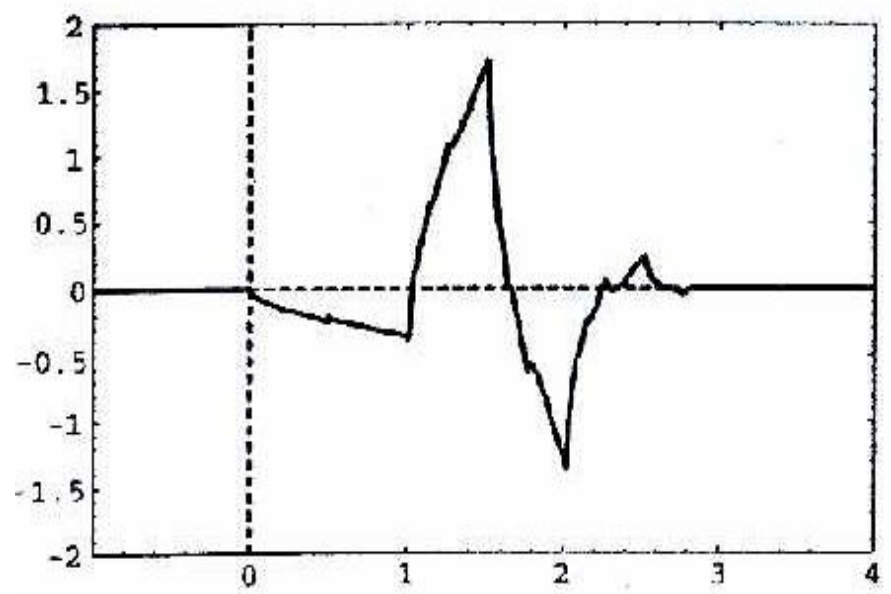

Fig.5b.Daubeches Wavelet function. For Rank 2 and Genus 2

\section{HIGH RESOLUTION WAVELET SPECTROGRAM}

The Morlet wavelet is adopted for general Gaussian based signal genesis events.

$$
h(t)=\exp \left(j \omega_{0} t\right) \cdot \exp \left(-t^{2} / 2\right)
$$

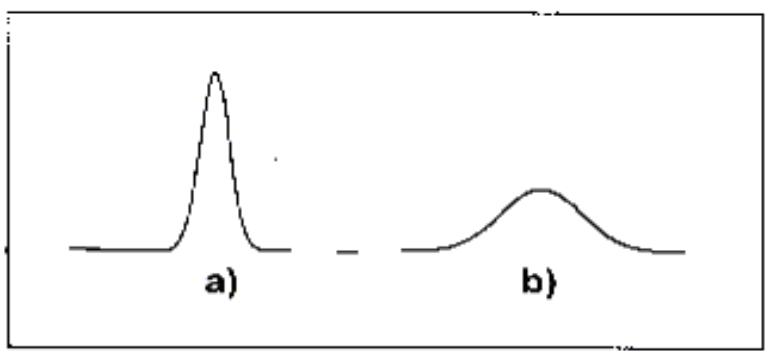

Fig.6. Shows the magnitude plot of the Morlet wavelet . a). Low value b). Increased scale value

It is actually a Gaussian-windowed complex sinusoid that is defined as following in the time and frequency.

A Morlet wavelet is having the function

$$
\psi(\mathrm{t})=C \exp \left(-t^{2} / \alpha^{2}\right) \operatorname{Cos}(5 t)
$$

with $\alpha=\sqrt{ } 2$ and $C=1$.

This contains the sinusoidal component and a Gaussian decay (the $-\mathrm{t}^{2}$ term). When this is used with a signal, the results are better than the windowed Fourier transform. In the time domain plot, the Morlet wavelet is shown with an adjustable parameter $m$ (wave number) of 6 . This is the smallest wave number that allows for an accurate signal reconstruction. The Gaussian's second order exponential decay results in very good time localisation. The real and complex functions are both shown.

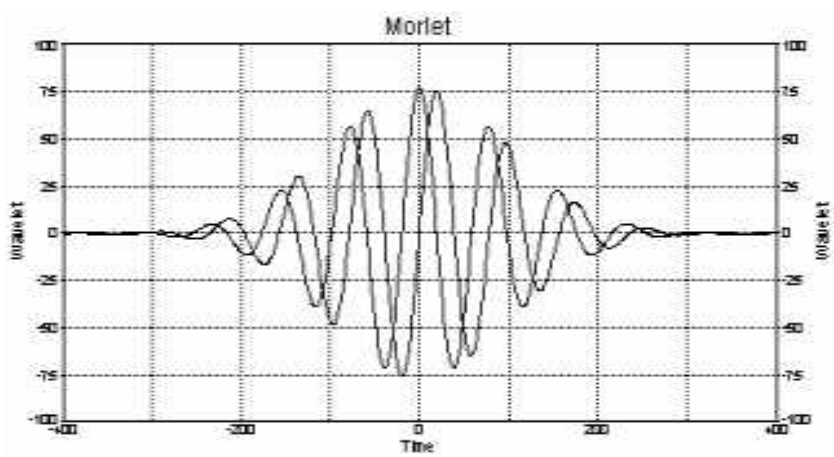

Fig.6c Gaussian-windowed complex sinusoid function

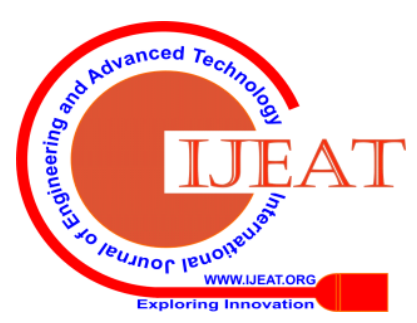




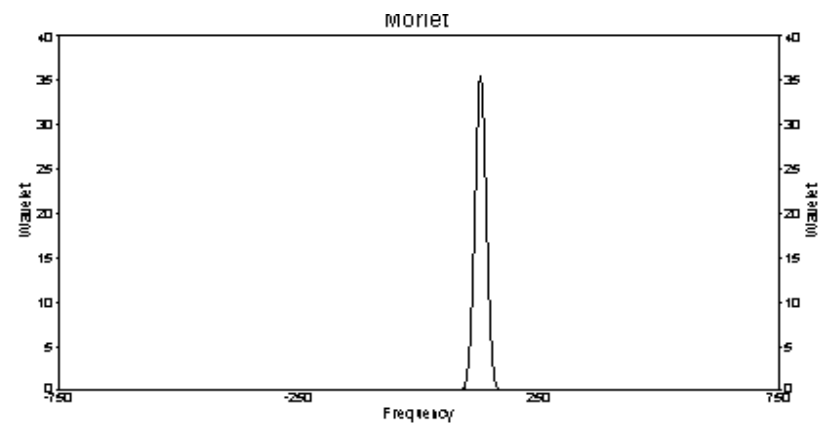

Fig.6d The frequency domain representation is a single symmetric Gaussian peak.

A single symmetric Gaussian peak and a sharp spectral peak of a sinusoid (fig.6d) ensures the frequency localization is very good.

The Morlet wavelet's adjustable parameter, the wave number, can vary from 6 to 20 in any signal. A Morlet wavelet with an adjustable parameter of 20 has a very different time domain representation.

In order to perform an undecimated WT decomposition, as in the bottom figure of 7, the FFT based method is to be employed. The Fourier transform of the continuous wavelet transform is

$$
F\{C W T(b, a)\}=(1 / \sqrt{a}) \phi^{*}\left(\frac{\omega}{a}\right) S(\omega)
$$

where $S(\varpi)$ if the FT of the signal $s(t)$ and $\phi *$ is that the wavelet scaling function $\phi(t)$. This can be precalculated and used for several values of $a$, the scaling factor.

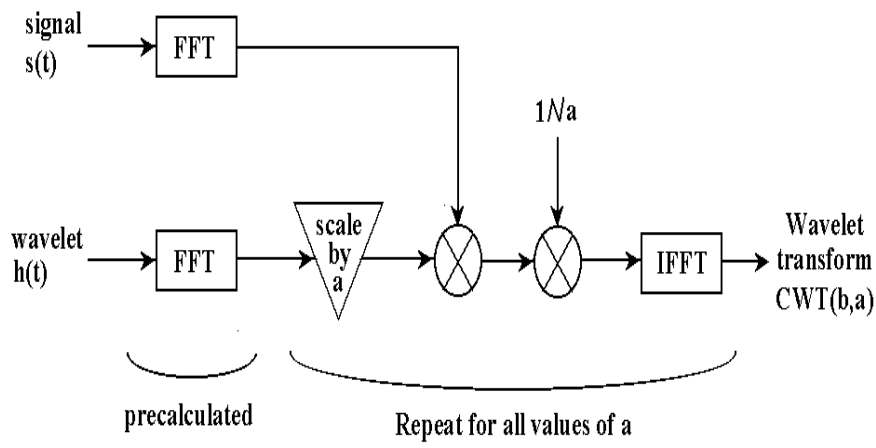

Fig.7. Shows the block diagram of this FFT based Discrete wavelet transform.

The FFT of the signal and wavelet, once calculated, is used for repeated scales, by multiply and inverse FFT calculations.

\section{A. NMR Signals}

For signals arising out of a large number of entities, the Gaussian based wavelet - the Morlet wavelet - is often prescribed. These arise in NMR signals in the R.F and magnetic filed environment by the millions of molecules of the chemical, whose energy is stochastically considered to be Gaussian distributed. Thus it is shown in fig. 8 how a Gaussian wavelet based (Gabor) transform [5] provides better resolution in the NMR spectrum of alcohol. The genesis of the signal is thus related to a Gaussian distribution and such a wavelet is ideal for its choice. The kind of inference we glean form such NMR spectra is of course the molecular groups $\mathrm{C}-\mathrm{H} 3, \mathrm{CH}-\mathrm{H}, \mathrm{OH}$ and so on. If the result of the wavelet used is able to show up these separately in the time- frequency grid, then, it is fit for this application.

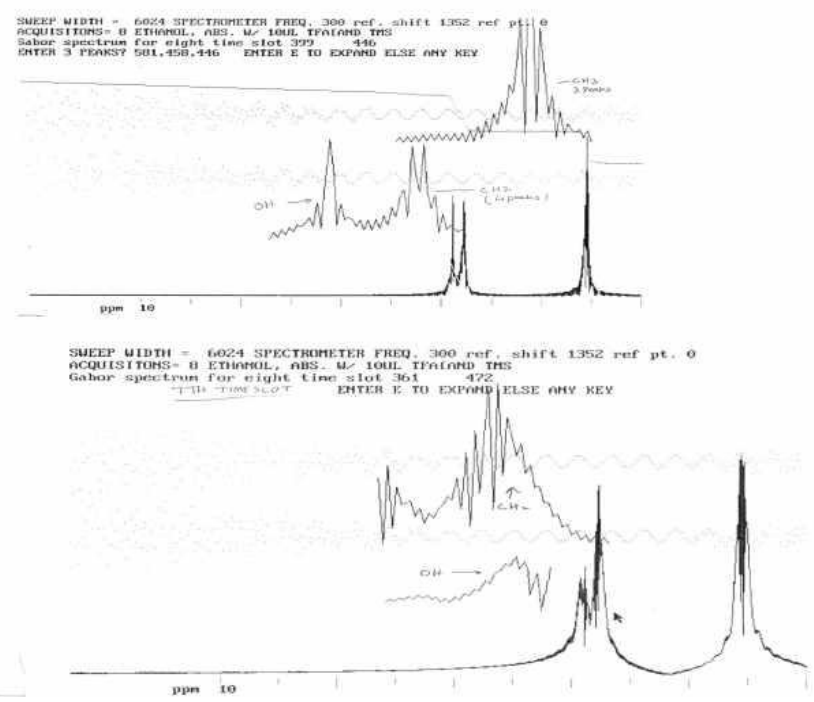

Fig. 8. Shows the wavelet (Gaussian) spectrum of the NMR signal (Ethonal). The peaks additionally Seen (bottom) are the ndicative of all the possible C-H couplings which are not seen in plain STFT spectrum (top).

\section{B. The Physiological ECG Signal}

Consider the case of the ECG signal being used for study by a wavelet decomposition. With the same Morlet, a study by a wavelet decomposition of the typical signal is shown in ig.9.[1]. There is much smear and neither the temporal nor frequency inference is any good.

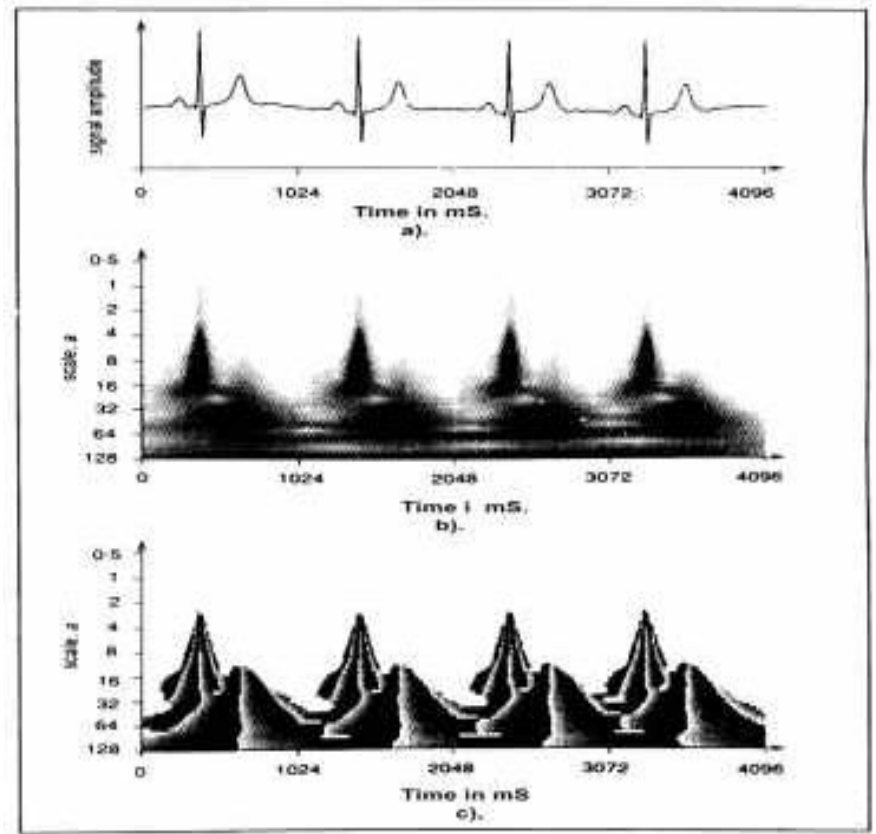

Fig.9 shows the ECG signal and the wavelet magnitude and phase spectrograms. Much smear and little inference are noteworthy

Clearly, this wavelet is unsuitable for this signal.

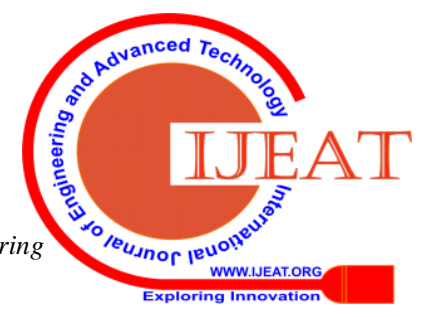




\section{Wavelet Transforms Applications and Interpretation Based On the Signal Genesis}

Hence, there is little inference available in this method. Of course, it clearly separates the high frequency components, giving rise the $\mathrm{R}$ wave conspicuously. It is ideally used for rate determination in noisy ecgs.

\section{Wavelet Imaging}

In some cases, wavelet decomposition has provided more than expected results [4]. In the case of 2- D WT applications for image data, the wavelet technique has provided a compression of the data. Because the wavelets in this case can suffer a certain amount of data loss, the method is used for compression as well as pattern recognition. The choice of the wavelets, in this case, need not relate to the genesis of the signal. In the case of detection of abnormal regions of interest (ROI), in medical radiograph data, the principle is to choose wavelets that provides the maximum contrast detail in the ROI.

\section{Denoising - Choice of Wavelet is useful}

Another important area is in the detection of signals with noise components brought about by the very nature of signal genesis. Ultrasound echo signals used in imaging for diagnostic medicine consist of backscattered echos creating interference patterns or 'speckle' noise, which can be modeled by either Rayleigh or Gaussian distribution. The Rayleigh density function $\mathrm{p}(\mathrm{x})$ of the echo envelope signal $\mathrm{X}$

$$
P(X)=\frac{X}{\sigma^{2}} \exp \left(-X^{2} / 2 \sigma^{2}\right)
$$

For these, the wavelet coefficients, after evaluation from the signal, are threshold limited. In a typical real time ultrasound imaging, after removing the speckles in this way, artifacts are corrected and edge enhancement is performed. In seismic analysis of tremor signals, the matching property with the signals is the criterion. Daubechie's D4 wavelet has been found to be indicative [5].

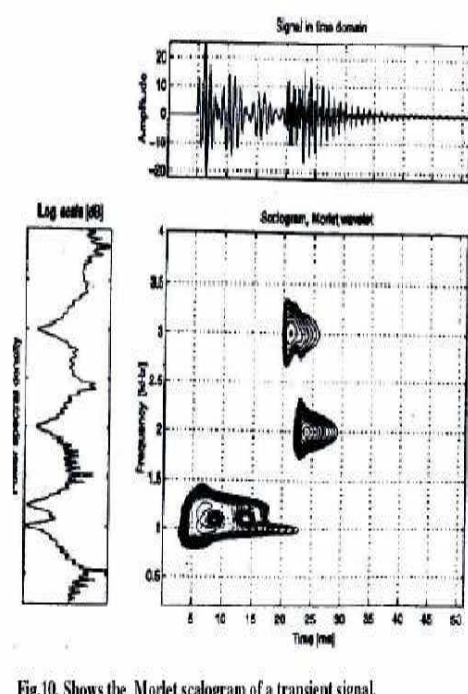

Fig.10. Shows the Morlet Scalogram

In power system transient signals, the analysis is more particular in regard to temporal resolution. Vibration of
Fig.10. Shows the Mortet scalogram of a transieut signal

worn contains in circuit breakers. In these, the Morlet wavelet has been tried with good results. Fig.10. Shows the Morlet calogram of a transient signal.

Singularity detection needs to identify the rising edges in a vibration signal, the timing of which are useful for condition assessment of circuit breaker contacts. If edges in the signal are to be detected, the first order differential maxima are the choice. To detect the local singularities, it is not necessary to use a wavelet with narrow frequency support, but vanishing of moments is crucial. To choose a wavelet $\psi(\mathrm{t})$ with an vanishing moments, it is first necessary to take a fast decaying function $\theta(t)$ and use its nth derivative by

$$
\psi(t)=(-1)^{n} d^{n} \theta(t) / d t
$$

and thereby find the CWT as

$$
\begin{aligned}
& C W T(a, b)=a^{n} D^{n}\left\{X^{*} \theta(a, b)\right\} \\
& \text { when } D^{n}=d^{n} / d t \text { and } \theta(a, b)=\frac{1}{\sqrt{a}} \theta(-t-b / a)
\end{aligned}
$$

In this method, ridge plots of vibrations can be shown on the CWT Spectrogram (Fig.11).

\section{EEG -WAVELET TRANSFORM BASED SIGNAL PROCESSING WITH TYPICAL EXAMPLES}

In the field of EEG for neuropathology and disorders, there are quite many references. Some aim at EEG noise reduction for a better visual observational diagnostics. A fast wavelet transform for EEG, with a view to real time instrument observation has been discussed. Multiresolution decomposition of EEG with a view to analyze local pathological patterns is very useful. Apart from denoising, another area has been in (automatic) identification of onset of spikes to aid in continuous patient monitoring. For a certain patient, from his past e.e.g. records, if a set pattern of spike and wave has been noted, a wavelet could be constituted and used for automatic matching with data from continuous monitoring. The choice of suitable wavelet for a signal is important so as to achieve for representing the results in a clear manner. For this purpose, one should know the signal genesis and its oscillatory components etc. The Fourier transform transforms the time domain information into frequency domain with an infinite number of frequencies of any signal. For reconstructing the signal, the inverse Fourier transform is used. We can reconstruct the signal back from the frequencies using the inverse transform due to orthogonality. Thus, it was possible to study events very much through a Fourier analysis all these days. Certain wavelets have orthogonality like the sinusoidal waves. With the ushering in of wavelets for many a signal analysis, based on the principles of multi-resolution analysis, the use of wavelets for every signal is now a commonplace work.

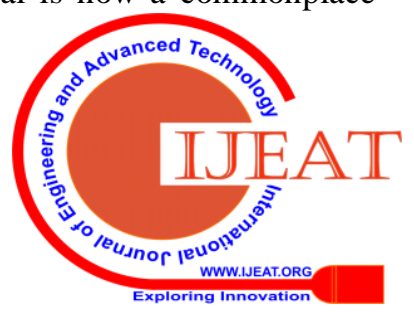


If a plain Fourier transform could give the necessary information for any signal, then, it is not worth the effort to try to portray the 3-dimensional plots of several wavelet decompositions. Really, wavelets give enormous information of the signal space as we have seen here; nevertheless, the available wavelets are based on mainly the mathematical foundations of the method rather than on the signal patterns.

The additional properties possessed by this would be equally important for wavelets such as:

1. Orthogonality with respect to unit weight to establish a relation to the physical energy

2. Symmetry of the absolute value

3. Good localization in physical space.
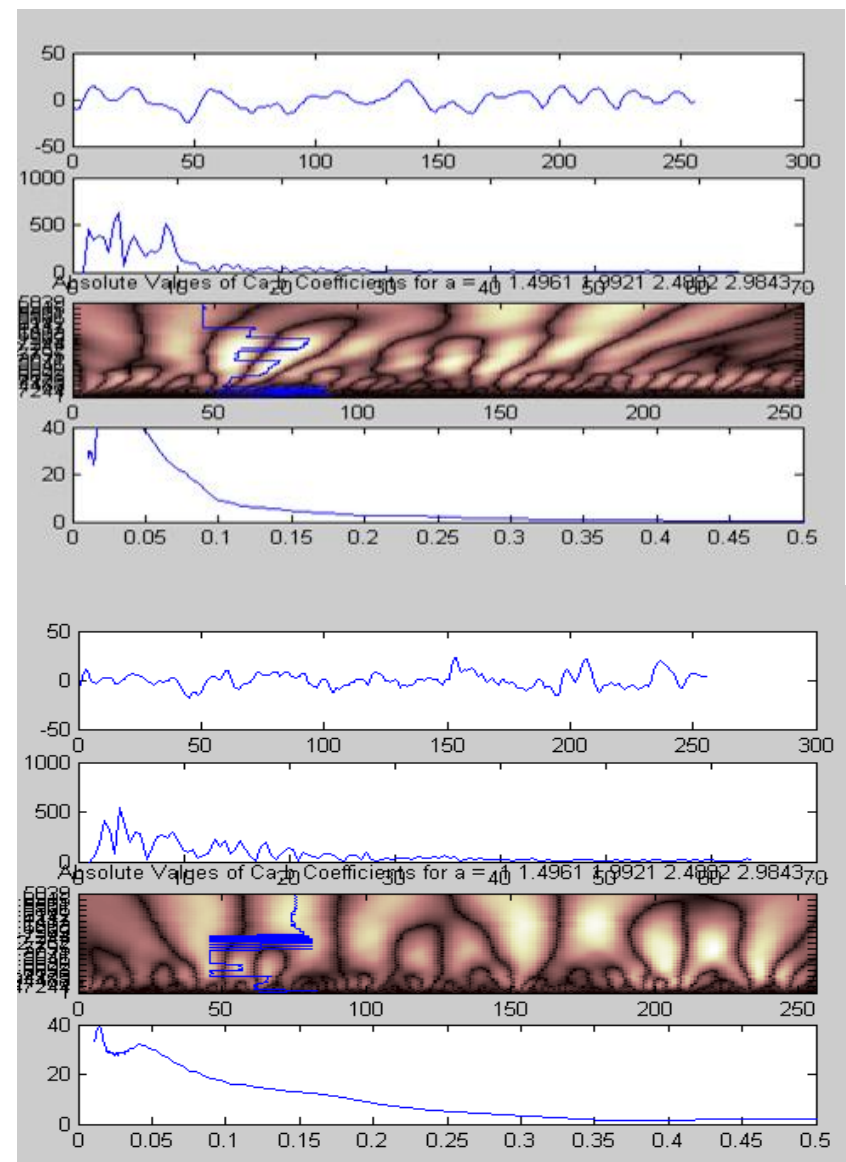

Fig.12. Examples of some records showing scalogram with optimal wavelet and also the line of maxima frequency plot.

The above records indicate how after finding the choice wavelet for a signal, the scalogram is useful for clear identification of the region of the high amplitude signal component and then the frequency plot shows the nature of the same (alpha, or lower frequency activity).

\section{CONCLUSION}

From a comparative study of the methods based on wavelet representations, it is clear that unless these wavelets are chosen properly for a particular type or nature of the signal, the results are not useful. It has been shown how the Gaussian - based wavelets are quite useful in signals derived from multitude of entities, but they fail in signals of limited number of vibrating elements.However, when it comes to a question of mere identification of an abnormal event amongst a large number of normal ones, the requirement is met by many of the nonlinear wavelets the D4 Daubechies, for example. We normally expect images to be decisive merely by their edges or contours; however, in diagnostic medicine, the details are elicited by a rather obtruse wavelet transform.

\section{REFERENCES}

1. P.M. Bentley and J.T.E. McDonnell, "Wavelet transforms: an Introduction," Electronics and Communication Engineering Journal, August 1994.

2. Howard.L, Resnikoff, Raymond O.Wells Jr., ' Wavelet Analysis, The scalable structure of information', Springer publications, New York, 2000.

3. D.Gopikrishna, Compression of EEG signals using Novel Wavelet ased techniques with application to Telemedicine, J1. of Inst.Sci., Bangalore, 2004.

4. A Petrosian and F.G. Meyer, 'Computer aided Radiographic Images' Chapter IV in wavelets in signal and image analysis.-., $\mathrm{K}$ luver Publishers,USA, Vol.19, 2000.

5. AA Petrosian and F.G. Meyer, 'An application of DWT in Geismic analysis', Chapter-16, Kluver Publishers, USA, Vol.19, 2000.

6. S.Ananthi and K.Padmanabhan, "Application of Gabor Spectrum for NMR signals", Oriental Journal of Chemistry, pp-1-12, 2000.

7. K.Padmanabhan ,S.Ananthi, R.Vijayarajeswaran, A practical Approach to Digital Signal Processing, New Age International Publishers, New Delhi, revised edition 2002.

8. Handbook of Mathematical Functions with Formulas, Graphs, and Mathematical Tables.-Chapter 8: Applied Mathematics Series 55 , Dover Publications. p. 332. ISBN 0-486-61272-4.

9. Speigel, Math. Handbook of Formulas and Tables, Schaum Series Publications, 1990.

10. Attewell. P.B. and Sandford M.R, On the Creiterion of failure for Geological Rock Crack Distribution, Symposium on Anisotropic Rocks, 1974

11. M. Do and W. Vetterli. The contourlet transform: an efficient directional multiresolution image representation. IEEE Trans. Im. Proc., 14(12):2091-2106, 2005.

12. D. Donoho and X. Huo. Beamlets and multiscale image analysis. In T. J. Barth, T. Chan, and R. Haimes, editors, Multiscale and multiresolution methods, volume 20 of Lecture Notes in Computational Science and Engineering, pages 149-196. Springer, 2002 .

\section{AUTHORS PROFILE}

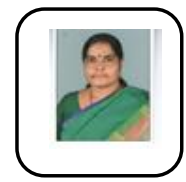

Dr.S.Ananthi, got her B.E. in ECE from the Anna University. She did M.Tech. at the Indian Institute of Science and later did Doctorate from University of Madras. She worked in the University as Professor and Head i/c of NSIT department till June 2018.After retirement, she is Professor, ECE in MVJCE. Her areas of specialization includes in DSP, Adv. Communicationand Biomedical telemetry. She was awarded the Env. TN award for the year 2004

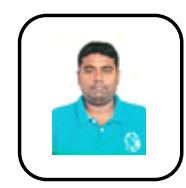

Dr Arun Ananthanarayanan has done his B.E from Anna University,M.S in Electrical Engineering from SUNY Buffalo, USA. His research interest includes Computer Networks,Signal processing ,Communication systems $\mathrm{He}$ completed his $\mathrm{PhD}$ from University of Madras . Having served as Assistant Professor for more than 7 years in reputed private Engineering college, currently he is working as Assistant Professor at MVJ College of Engineering Bangalore. 


\section{Wavelet Transforms Applications and Interpretation Based On the Signal Genesis}

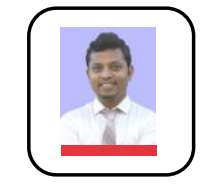

Sivakannan Subramani completed his $\mathrm{PhD}$ from Annamalai University under the Faculty of Electronics and Instrumentation Engineering, Chidambaram, India. $\mathrm{He}$ is working as an Assistant Professor at the Department of Electronics and Communication Engineering, MVJ College of Engineering, Bengaluru, Karnataka. His research interest includes image and video processing, VLSI design, embedded systems and wireless communication. He has published 12 papers in international journals and ten papers in international/national conferences. $\mathrm{He}$ has around eight years of experience in both teaching and industry.

Mr.Prahanth has completed B.E in Electronics and communication Engineering from Karnataga and M.Tech in Signal Processing from Reva College of Engineering, Bangalore. Presently, he is working as Assistant Professor, ECE Department, in MVJ College of Engineering, Bangalore. His expertise includes Signal processing techniques, Embedded applications. 